Neurosurg Focus 19 (3):Intro, 2005

\title{
Stem cell opportunities for neurosurgery
}

\author{
Charles Y. LiU, M.D., Ph.D. And Alfredo Quinones-Hinojosa, M.D. \\ Department of Neurological Surgery, University of Southern California Keck School of Medicine, Los \\ Angeles, California, and Department of Neurosurgery, Johns Hopkins University, Baltimore, \\ Maryland
}

Perhaps the most important reason that stem cell development is so appealing to neurosurgeons can be found in the statement: "the adult human brain, in contrast to other organs such as skin and liver, lacked the capacity for selfrepair and regeneration." This fundamental axiom of neuroscience has put severe limitations on treatment options for patients with neurological diseases. In fact, the progressive minimalism that has characterized the evolution of neurosurgery has largely been fueled by the desire to avoid damage to the nervous system during surgery. In addition, prevention of deficits has been the achievable alternative to the restoration of function after damage. However, in contrast to what has been believed for much of the last century, the adult nervous system may be capable of repairing itself. We now know that stem cells exist in the adult brain as well as in the embryo and that these cells can be isolated and transplanted, after which they survive, differentiate, migrate, and appear to integrate. Stem cells have been shown to produce positive therapeutic effects in animal models and in a few human trials. However, the exact mechanism of this positive effect is as yet unclear, and perhaps produced more by secretion of neurotrophic factors than by actual integration into the defective neural architecture. We know that stem cells in neurogenic areas of the brain can be induced to activate in response to an insult such as trauma or ischemia. There is now growing evidence that stem cells may play a role in neoplasia and cancer, where tumors are derived from the loss of control over tumor stem cells. We are currently moving toward the elucidation of the functional aspects of stem cells in addition to their histology.

The true promise of stem cells is that, in contrast to what has been believed for most of the history of neurosurgery, stem cells may ultimately allow repair of neurological injury rather than merely its prevention. This dramatically broadens the spectrum of therapeutic possibilities. Indeed, the potential targets for restorative neurosurgery cover the entire spectrum of neurological disorders, including stroke (which is the second biggest killer of Americans after heart disease), neurodegenerative disorders (the number of afflicted patients overwhelm most other neurosurgical patients added together), traumatic injury, tumors, spinal cord injury, peripheral nerve injury, developmental disorders, and even spinal degenerative disorders. In this issue of Neurosurgical Focus, such topics with immediate relevance to neurosurgeons and the neurosurgical field are covered, including induction of endogeneous stem cell proliferation after nervous system injury, the stem cell microenvironment, spinal cord injury, stem cell therapy for degenerative spinal disc disease, and the treatment of malignant brain tumors. 